\title{
Repair of Acute Type B Aortic Dissection Complicated by Aortic Rupture with Debranching Thoracic Endovascular Aortic Repair and Left Subclavian Artery Occlusion Using Amplatzer Vascular Plug II
}

\author{
Yasunori lida, MD, PhD, ${ }^{1}$ Tsutomu Ito, MD, PhD, ${ }^{1}$ Sachiko Hayashi, MD, ${ }^{1}$ Tatsuo Takahashi, MD, ${ }^{1}$ \\ Takahiko Misumi, MD, PhD, ${ }^{1}$ Takashi Hachiya, MD, ${ }^{2}$ and Hideyuki Shimizu, MD, PhD ${ }^{2}$
}

An 88-year-old man with severe chest pain and syncope was admitted to our hospital. Contrast-enhanced computed tomography $(\mathrm{CT})$ revealed acute type $\mathrm{B}$ aortic dissection with rupture. Considering age and operative risk, we performed emergency thoracic aortic endovascular repair with twodebranching of the left common carotid and left subclavian arteries. To prevent type II endoleak, we used Amplatzer Vascular Plug (AVP) II for left subclavian artery embolization. Postoperative contrast-enhanced CT showed no type II endoleak and rupture site exclusion. As postoperative persistent blood flow to the primary entry or rupture site causes re-rupture, AVP II was crucial in preventing type II endoleak.

Keywords: aortic dissection, endovascular procedures

\section{Introduction}

Aortic rupture is one of the catastrophic complications of acute type $B$ aortic dissection (ABAD). In patients with this type of rupture, the role of thoracic endovascular aortic repair (TEVAR) has been debated. In the International Registry of Acute Aortic Dissection review, the in-hospital mortality rate in patients undergoing surgical repair of type B aortic dissection was $29.3 \%$, with a higher rate of $62.5 \%$ in patients with aortic rupture. ${ }^{1)}$

We successfully treated an 88-year-old man who suffered from ABAD and aortic rupture by debranching TEVAR and left subclavian artery (LSA) embolization

${ }^{1}$ Department of Cardiovascular Surgery, Saiseikai Yokohamashi Tobu Hospital, Yokohama, Kanagawa, Japan ${ }^{2}$ Department of Cardiovascular Surgery, Keio University, Tokyo, Japan

Received: May 6, 2015; Accepted: May 27, 2015

Corresponding author: Yasunori Iida, MD, PhD. Department of Cardiovascular Surgery, Saiseikai Yokohamashi Tobu Hospital, 3-6-1 Shimosueyoshi, Tsurumi-ku, Yokohama, Kanagawa 230-8765, Japan Tel: +81-45-576-3000, Fax: +81-45-576-3586

E-mail:y.iida@vanilla.ocn.ne.jp using Amplatzer Vascular Plug (AVP) II to prevent type II endoleak and re-rupture.

\section{Case}

An 88-year-old man with a history of hypertension was admitted to our hospital because of the sudden onset of severe chest pain and temporary syncope. His heart rate and blood pressure were $110 \mathrm{bpm}$ and 82/40 $\mathrm{mmHg}$. Refractory chest pain and vital deterioration continued despite analgesic administration and transfusion. Contrast-enhanced computed tomography (CT) showed type B aortic dissection with aortic rupture (Fig. 1A and 1B). CT angiogram revealed the primary entry tear in the distal arch and contrast medium leakage to the mediastinum (Fig. 1C).

Under general anesthesia, arch vessel debranching using a ringed expanded polytetrafluoroethylene graft (W.L. Gore \& Associates, Flagstaff, AZ) was made and anastomosed end-to-side to the subclavian arteries and end-toend to the carotid artery, with proximal stump ligation. Two Valiant stent grafts, $38 \mathrm{~mm} \times 150 \mathrm{~mm}$ and $34 \mathrm{~mm} \times$ $200 \mathrm{~mm}$ (Medtronic, Santa Rosa, CA) were deployed from the orifice of the left common carotid artery to the descending aorta. Next, LSA occlusion was performed via the LSA distal site; the LSA was catheterized with a straight 6-Fr guiding sheath (Destination; Terumo Corp., Tokyo, Japan), proximal to the left vertebral artery. A 14-mm Amplatzer Vascular Plug (AVP) II (St. Jude Medical Inc., St. Paul, $\mathrm{MN}$ ) was placed through the guiding sheath, confirming the correct position, and deployed by turning the delivery wire (Fig. 2A). Digital subtraction angiogram immediately after plug deployment showed slight residual type II endoleak (Fig. 2B), which eventually was not recognized in the postoperative follow-up CT.

The postoperative course was uneventful. CT angiogram on postoperative day 5 demonstrated patent debranching bypasses and exclusion of the aortic lesion without any further endoleak (Fig. 3A-3C). 

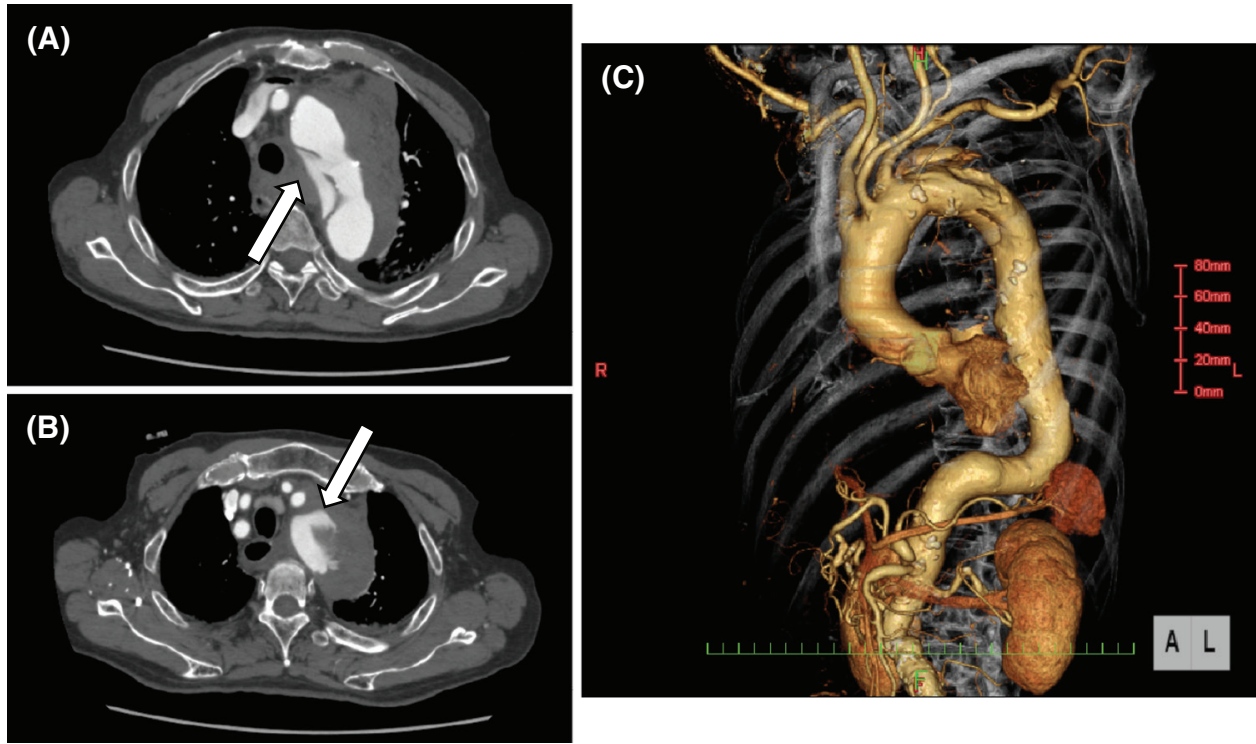

Fig. 1 (A, B) Preoperative contrast-enhanced CT showing type B aortic dissection with the primary entry tear located in the distal arch (arrow) and an aortic rupture with contrast medium leakage to the mediastinum (arrow). (C) CT angiogram showing the entry site and rupture site together. CT: computed tomography
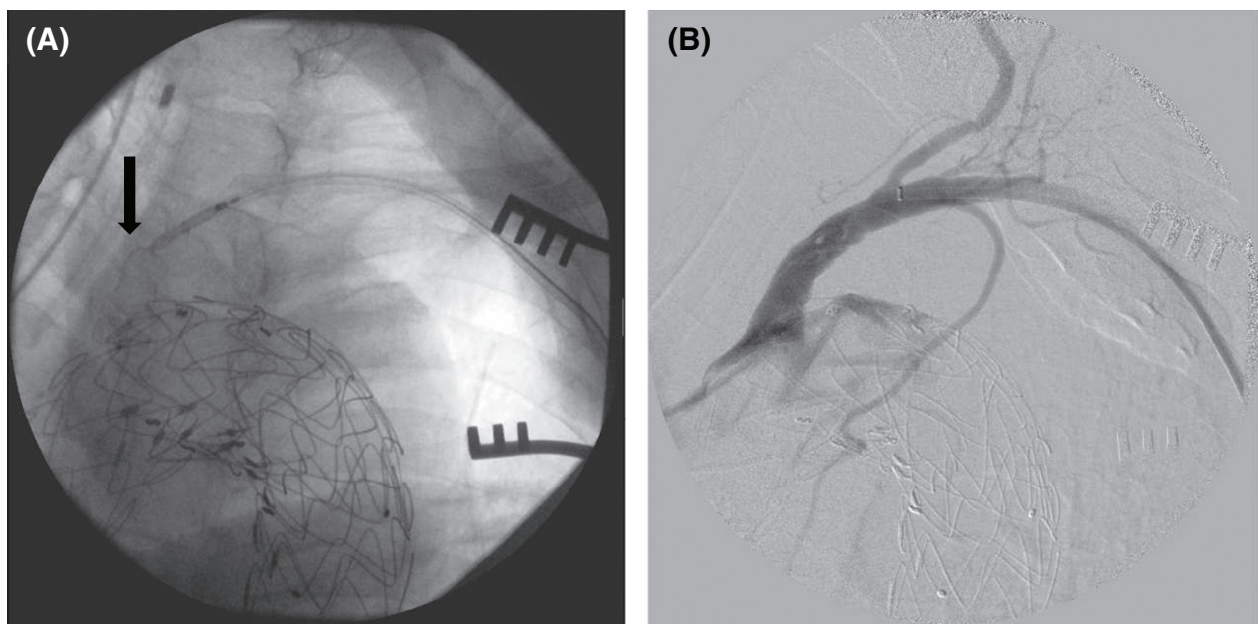

Fig. 2 (A) Intraoperative X-ray fluoroscopy showing a semi-deployed AVP II (arrow). (B) Digital subtraction angiogram immediately after plug deployment showing slight residual type II endoleak, which eventually was not recognized in the follow-up CT. СT: computed tomography

\section{Discussion}

The management of acute complicated type B aortic dissection remains a challenging issue. Trimarchi et al. demonstrated that conventional surgical repair for $\mathrm{ABAD}$ is still associated with significant morbidities, with an overall in-hospital mortality rate of $29.3 \%$. Moreover, for patients presenting with aortic rupture, the in-hospital mortality rate was $62.5 \% .^{1)}$ In contrast, Szeto et al. ${ }^{2)}$ reported the effectiveness of TEVAR for ABAD, with a 30 -day mortality rate of $2.8 \%$. Other studies investigating the effectiveness of TEVAR in acute complicated type B aortic dissection have been published. Nienaber et al. ${ }^{3)}$ reported the absence of morbidity or TEVAR-related complications and no operative deaths in 11 patients who underwent TEVAR for ABAD with contained rupture. Chen et al. ${ }^{4)}$ reported a technical success rate of $100 \%$ and a 30-day mortality rate of $4.4 \%$ in 23 patients who underwent emergency TEVAR for ABAD.

For aortic dissection complicated with aortic rupture, not only coverage of the primary entry tear but also the exclusion of blood flow from the rupture site is indispensable. 

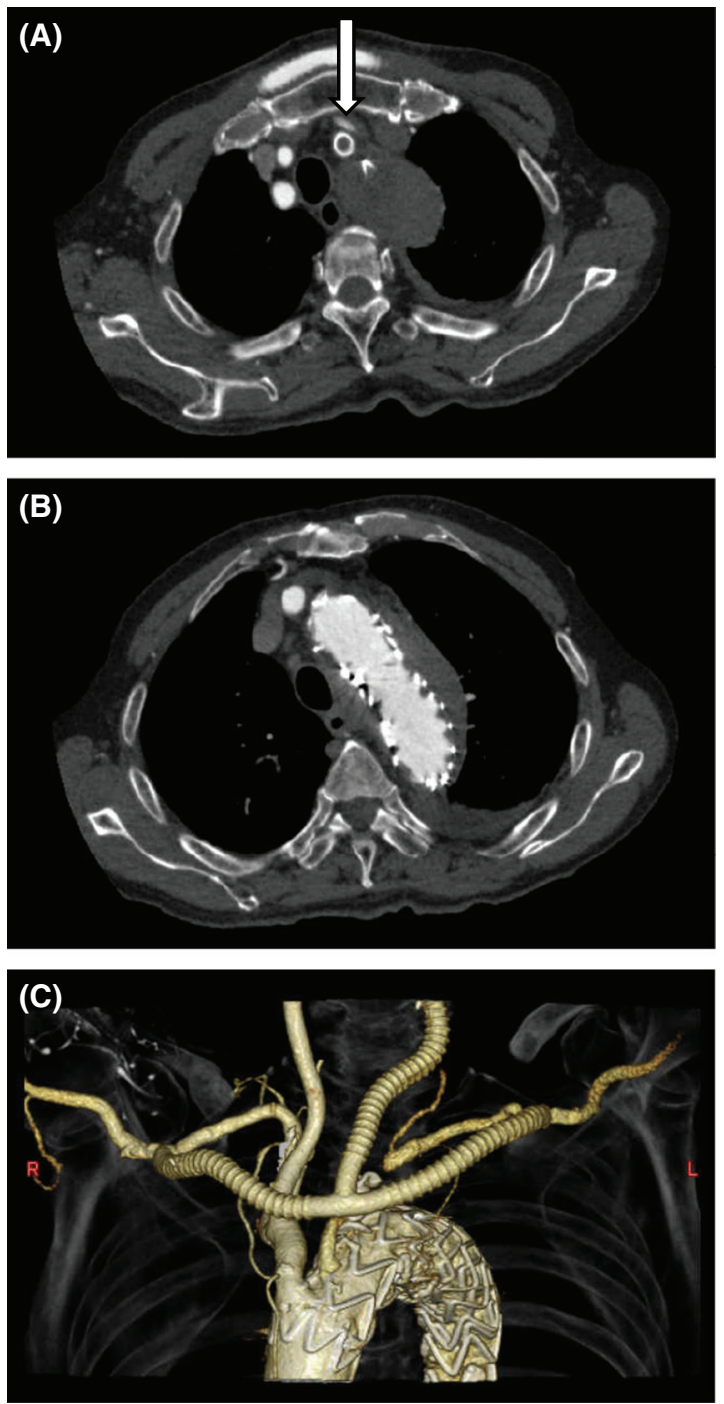

Fig. 3 (A) CT scan on postoperative day 5 demonstrated an embolized LSA orifice (arrow). (B, C) Patent debranching bypasses were recognized and exclusion of the aortic lesion with no endoleak was achieved. CT: computed tomography; LSA: left subclavian artery

In particular, when type II endoleak from the LSA persists, this indicates continued blood flow from the LSA to the rupture site, which leads to re-rupture or incomplete exclusion of the lesion. To prevent type II endoleak, we used AVP II intraoperatively, achieving complete blockage of blood flow from the LSA. Chaudhuri et al..$^{5)}$ used first-generation AVP (St. Jude Medical Inc.) to embolize a partially uncovered LSA post-TEVAR to prevent thromboembolism of the upper limbs or vertebral artery, achieving excellent results. Meyer et al. ${ }^{6}$ reported the efficacy of second-generation AVP for the treatment of type II endoleak from the LSA post-TEVAR. In the present case, we were able to completely prevent type II endoleak, which usually leads to re-rupture owing to residual blood flow from the LSA orifice to the rupture site, using AVP II. To the best of our knowledge, this is the first case of ABAD with aortic rupture successfully treated by debranching TEVAR in combination with AVP II to prevent type II endoleak in the rupture site, avoiding the catastrophic postoperative re-rupture.

\section{Conclusion}

We successfully treated an 88-year-old man who suffered from $A B A D$ and aortic rupture by emergency debranching TEVAR and LSA embolization using AVP II to prevent type II endoleak and re-rupture. For conditions involving ABAD complicated by aortic rupture, treatment using TEVAR in combination with LSA occlusion with AVP II may be effective.

\section{Acknowledgement}

We are indebted to Dr. Edward F. Barroga, Associate Professor and Senior Medical Editor of Tokyo Medical University for editing the manuscript.

\section{Disclosure Statement}

Yasunori Iida and other co-authors have no conflict of interest.

\section{References}

1) Trimarchi $S$, Nienaber CA, Rampoldi V, et al. Role and results of surgery in acute type $\mathrm{B}$ aortic dissection: insights from the International Registry of Acute Aortic Dissection (IRAD). Circulation 2006; 114(1 suppl): I357-64.

2) Szeto WY, McGarvey M, Pochettino A, et al. Results of a new surgical paradigm: endovascular repair for acute complicated type B aortic dissection. Ann Thorac Surg 2008; 86: 87-93; discussion 93-4.

3) Nienaber CA, Ince H, Weber F, et al. Emergency stent-graft placement in thoracic aortic dissection and evolving rupture. J Card Surg 2003; 18: 464-70.

4) Chen S, Yei F, Zhou L, et al. Endovascular stent-grafts treatment in acute aortic dissection (type B): clinical outcomes during early, late, or chronic phases. Catheter Cardiovasc Interv 2006; 68: 319-25.

5) Chaudhuri A, Tibballs J, Nadkarni S, et al. Digital embolization due to partially uncovered left subclavian artery post TEVAR: management with amplatzer vascular plug occlusion. J Endovasc Ther 2007; 14: 257-9.

6) Meyer C, Probst C, Strunk H, et al. Second-generation Amplatzer Vascular Plug (AVP) for the treatment of subsequent subclavian backflow type II endoleak after TEVAR. Cardiovasc Intervent Radiol 2009; 32: 1264-7. 